\title{
Sistema de monitoreo usando tecnología XBee y GSM para la supervisión del clima en la producción de plátano
}

\author{
Mónica M. Miranda-Ramos*, Alex A. Ortiz y Lissette A. Moreno \\ Universidad Politécnica Salesiana, Fac. de Ingeniería, Dpto. de Ing. Electrónica., Guayaquil-Ecuador. \\ (correo-e: mmiranda@ups.edu.ec; alex.ortiz.p.89@gmail.com; lissette_17_mb@hotmail.com) \\ * Autor a quien debe ser dirigida la correspondencia.
}

Recibido May. 18, 2020; Aceptado Jul. 15, 2020; Versión final Ago. 17, 2020, Publicado Dic. 2020

\begin{abstract}
Resumen
En este artículo se presenta el diseño de un sistema de monitoreo que muestra las condiciones climáticas en un cultivo de plátano con la generación de alertas en caso de detectar variables climáticas fuera del rango óptimo de producción. La mayoría de los cultivos de plátano son controlados por un operador manual, elevando costos de operación. Se utilizan sensores electrónicos y tecnología inalámbrica de comunicación (XBee y GSM) utilizando un sistema embebido Arduino e interpretado en el software Qt Creator con interacción a una base de datos desarrollada en MySQL. El sistema está compuesto por dos estaciones de campo donde se encuentran los sensores para lectura de las variables climáticas y una estación de monitoreo para supervisión fuera de la zona agrícola. Se concluye que el sistema de monitoreo presentado permite supervisar el cultivo de plátano vía remota, teniendo acceso a un historial de registros del clima y valores fuera de rango, lo que posibilita evitar pérdidas de producción agrícola.
\end{abstract}

Palabras clave: XBee; Arduino; Qt Creator; MySQL; variables climáticas; cuidado del banano; sensores iot

\section{Monitoring system using XBee and GSM technology for climate supervision in plantain production}

\begin{abstract}
The present study presents the design of a monitoring system that shows climate conditions in a plantain crop by generating alerts of climate variables detected outside the optimal production range. Most plantain crops are controlled by a manual operator, which raises operational costs. The monitoring system uses electronic sensors and wireless communication technologies (XBee and GSM) using an embedded Arduino system. This is interpreted in the QT Creator software by interacting with a database developed in MySQL. The system is composed of two field stations where climate variables reading sensors are located. A monitoring station for supervision is located outside the agricultural area. It is concluded that the monitoring system presented here allows the user to monitor plantain crops remotely and to have access to a history of climate records and outof-range values that permit avoiding crop production losses.
\end{abstract}

Keywords: XBee; Arduino; Qt Creator; MySQL; climate variables; banana care; iot sensors 


\section{INTRODUCCIÓN}

La composición de los alimentos puede verse influenciada por factores ambientales como las condiciones climáticas y del suelo (Granato et al., 2016). El cambio climático tiene un gran impacto en la subsistencia o en los pequeños agricultores (Sujakhu et al., 2016). Las plantaciones comerciales a gran escala y los agronegocios asociados, se verán afectados de manera similar (Machovina y Feeley, 2013). La producción de cultivos tiene diferentes impactos dependiendo de la elevación, el tipo de cultivo y temporada de crecimiento (Poudel y Kotani, 2013). Por ejemplo, es probable que el cambio climático genere cambios geográficos en los cultivos y la idoneidad de la tierra, dando como resultado cambios en la aparición de plagas y enfermedades de los cultivos (Cintra et al., 2008), estos impactos pueden suponer una gran amenaza para diferentes cultivos comerciales. Si no se abordan los posibles efectos del cambio climático a través de cambios apropiados en las prácticas y técnicas agrícolas, luego disminuirán los rendimientos, las relaciones comerciales y la seguridad alimentaria local y nacional (Nelson et al., 2009).

Un producto de particular interés para estudiar esta relación es el plátano (Musa spp.). A nivel mundial, el plátano es una de las frutas más populares. El valor nutricional con su bajo contenido en grasa, azúcar, pero más alto en minerales, fibra y vitaminas hace que el plátano sea popular entre los consumidores (Singh et al., 2016). La mayoría de los plátanos de exportación se cultivan en las regiones de América Latina y el Caribe debido a las condiciones ambientales favorables y la proximidad de los mercados (Aurore et al., 2009).

Aunque el precio minorista de los plátanos no es alto, todavía hay un gran rendimiento y consumo de plátanos, así como miles de agricultores, minoristas y consumidores involucrados en el comercio mundial de plátanos. De este modo, lo convierten en una mercancía vital. Las exportaciones de plátano son uno de los ingresos económicos clave en la mayoría de los países centroamericanos y del sudeste asiático. La identificación del origen geográfico de los plátanos es necesaria para proteger los intereses de los agricultores (Van Rijn et al., 2019). Actualmente, el plátano se cultiva en más de 120 países tropicales y subtropicales y es una de las mayores fuentes de ingresos para pequeños y medianos agricultores (Soares et al., 2013). Los plátanos son un vehículo adecuado para estudiar la relación entre los factores geográficos y la composición de los alimentos, dada la variación en las condiciones de cultivo y su importancia en el comercio mundial (Wang et al., 2020).

Con el desarrollo de la tecnología de la información en los últimos años, el sistema agrícola ha recibido cada vez más atenciones. El sistema inteligente de simulación de agricultura es una rama importante de la agricultura moderna, que ayudará a los investigadores a completar el experimento agrícola, el crecimiento de los cultivos en tiempo real y la predicción del rendimiento (Jiayu et al., 2015). La agricultura es un área prometedora donde los dispositivos loT (internet de las cosas) pueden aliviar muchos problemas y proporcionar soluciones prometedoras. Cuando los dispositivos loT y las redes inalámbricas de sensores (WSN) se utilizan en la agricultura, se pueden aplicar técnicas agrícolas avanzadas que se conoce como agricultura de precisión (PA), para obtención, monitoreo y análisis de variables climáticas (Muñoz et al., 2019). Una de las características más importantes de la loT ha sido la comunicación máquina-máquina (M2M), en la cual todos los dispositivos involucrados pueden enviar mensajes entre sí, sin necesidad de intervención humana y que puede ser implementada mediante hardware libre, aumentando la eficiencia y reduciendo los costos (Luna et al., 2020).

Los dispositivos loT se pueden usar en sistemas de monitoreo que consisten en nodos que interactúan en el entorno utilizando sensores para recopilar información en tiempo real y transmitirla a una sala de control para su posterior procesamiento (Sadowski y Spachos, 2020). Sin embargo, en cada sistema de monitoreo, el consumo de energía es una de las principales preocupaciones para que el sistema funcione correctamente. Si un sensor deja de transmitir, faltarían datos sobre su área de monitoreo y el sistema ya no tendría información precisa. La energía solar es un enfoque prometedor, ya que está fácilmente disponible y se puede aprovechar para permitir que los sensores funcionen durante un período de tiempo más largo (Khan et al., 2018).

En este estudio, se realiza un sistema de monitoreo con el fin de que el agricultor optimice la producción del cultivo del plátano, mediante la medición automática de los parámetros ambientales del mismo, que permite determinar los niveles óptimos para el buen desarrollo agrícola, brindando al agricultor la facilidad de acceder a equipos tecnológicos y conocer de forma remota el estado del microclima generado en su cultivo para optimizarlo o prevenir la generación de enfermedades que puedan afectar al mismo. El sistema consta de dos estaciones de campo para monitoreo agrícola del cultivo y una estación base de monitoreo con conexión inalámbrica de XBee y GSM. Dicha información será interpretada por un software que tiene interacción con una base de datos para registro y consulta de forma remota, con una interfaz amigable para el usuario. Al concluir, se describen los resultados de las condiciones climáticas capturadas por las estaciones de campo en el cultivo de plátano. 


\section{METODOLOGÍA}

En este artículo se explica el diseño de un sistema de monitoreo de las condiciones climáticas del cultivo de plátano, con una red inalámbrica que conecta una red de sensores electrónicos para monitorear los parámetros ambientales con el objetivo de mejorar la producción del cultivo de plátano.

\section{Descripción del sistema}

El sistema consta de dos estaciones de campo, las cuales están ubicadas una en cada hectárea y separadas a la distancia óptima de comunicación entre ellos. Las estaciones de campo fueron nombradas como: estación de campo A y estación de campo B. La Fig. 1 muestra el esquema general del proyecto donde se puede ver que en las estaciones de campo se captura las variables climáticas a través de sensores electrónicos de: temperatura, humedad relativa, humedad del suelo, precipitación de lluvias, intensidad lumínica, velocidad del viento y radiación ultravioleta.

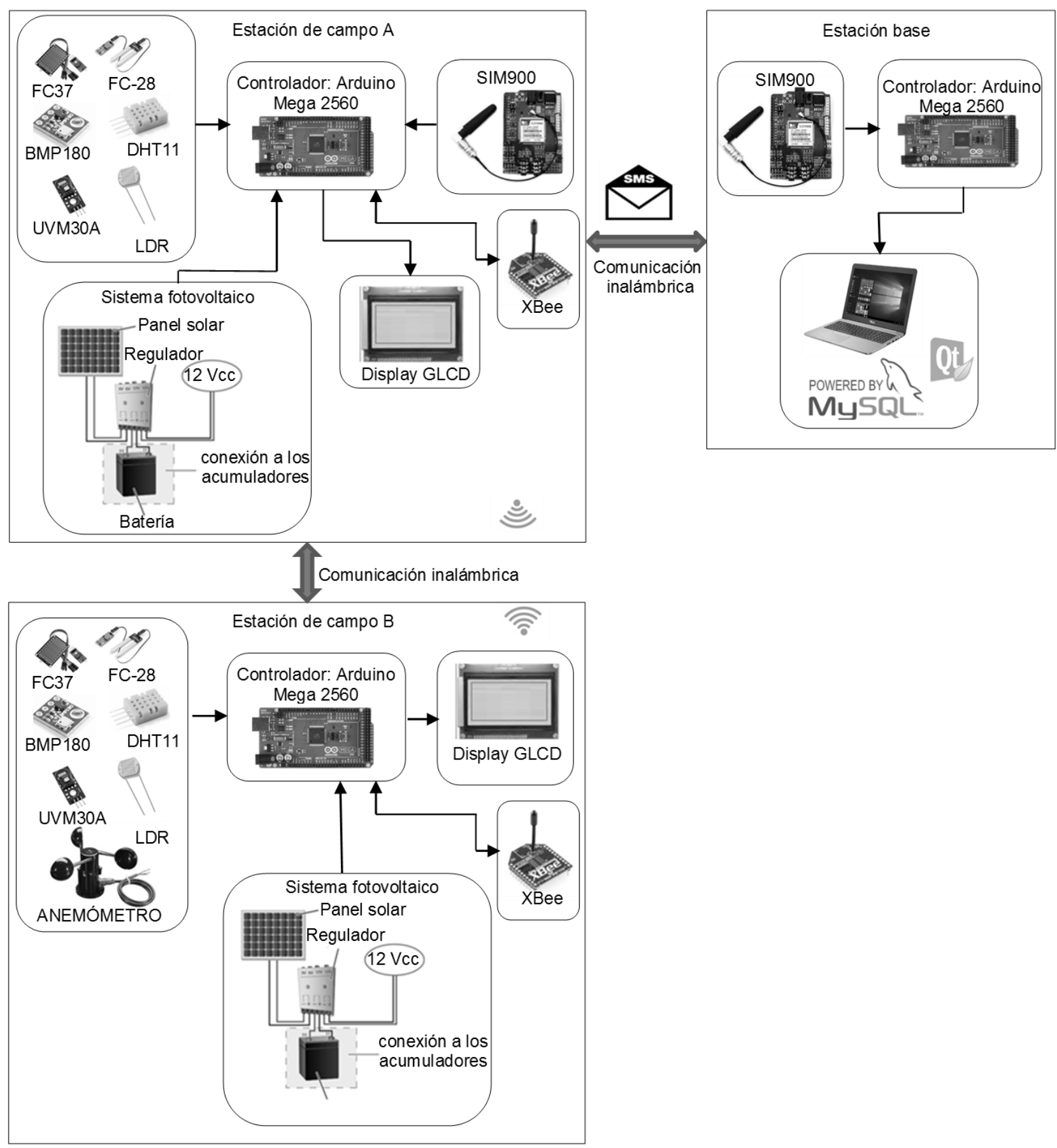

Fig. 1: Esquema general del proyecto para el monitoreo del clima en el cultivo de plátano 
Para la lectura de las variables obtenidas por los sensores electrónicos se utiliza el microcontrolador Arduino Mega 2560, transmitiendo información entre sí de forma inalámbrica a través de módulos XBee. La estación base actúa como la central de monitoreo del sistema; es la encargada de recibir la información adquirida en las estaciones de campo a través de dos módulos SIM900 por medio de un mensaje de texto para procesarlos y enviarlos a un computador para interpretación mediante el software desarrollado en Qt Creator, en conjunto con una base de datos desarrollada en MySQL, en el gestor de base de datos phpMyAdmin, donde se crea las tablas especificando los campos, tipos de datos y tamaño de la variable. Además, se puede visualizar señales de alerta cuando las condiciones climáticas capturadas se encuentren fuera del rango óptimo de producción. También se utiliza un módulo de display tipo GLCD para mostrar en la parte externa de cada módulo la información capturada. La Fig. 2 muestra el módulo de monitoreo mismo que utiliza los siguientes componentes que permiten analizar el comportamiento de las condiciones ambientales del cultivo que ayuda a los investigadores a completar el experimento agrícola, el crecimiento de los cultivos en tiempo real y la predicción del rendimiento.

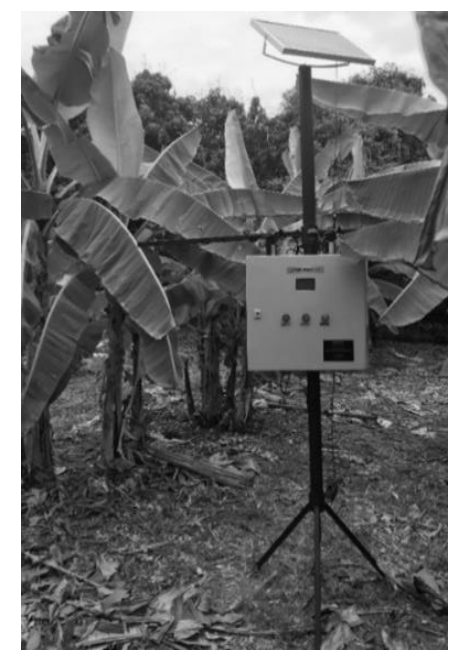

Fig. 2: Módulo de la estación de campo A del cultivo de plátano

Arduino mega 2560: es una placa de desarrollo basada en el microcontrolador ATmega2560. Tiene 54 entradas/salidas digitales (de las cuales 15 pueden ser usadas como salidas PWM), 16 entradas analógicas, 4 UARTs, un cristal de $16 \mathrm{MHz}$, conexión USB, jack para alimentación DC, conector ICSP, y un botón de reinicio. (Rafiq et al., 2018).

Módulo XBee: el módulo XBee es un transceptor inalámbrico que tiene un rango amplio de operación, en términos de distancia. La base de operación de XBee es generalmente un protocolo que se implementa completamente con el propósito de comunicación. Ofrece atributos esenciales que son imprescindibles para las comunicaciones de red prácticas en cualquier red que funcione de forma inalámbrica. Las características importantes como el direccionamiento y los reconocimientos ayudan a la eficiencia y seguridad de los datos que se entregarán en un nodo específico (Bhat et al., 2015).

Sensor de temperatura y humedad DHT11: tiene un módulo de medición de humedad, un termistor y un circuito integrado en la parte posterior de la unidad del sensor. El módulo de medición de humedad consta de dos electrodos. Entre los dos electrodos se encuentra un sustrato que es capaz de retener la humedad. El cambio en la humedad altera la conductividad del sustrato que retiene la humedad y al mismo tiempo cambia la resistencia. El circuito integrado procesa el cambio en la resistencia y se mide el valor de humedad. Por otro lado, el cambio de temperatura cambia la resistencia del termistor que es procesado por el circuito integrado y los resultados de la calibración en un valor de la temperatura (Bhadani y Vashisht, 2019).

Sensor de presión atmosférica y altitud BMP180: es un sensor de presión de aire, mide la presión absoluta alrededor del sensor y varía según el clima y la altitud. Dependiendo de cómo usar los datos, el BMP180 puede usarse para monitorear cambios climáticos, mediciones de altura u otras tareas que requieren lecturas de presión precisas. La frecuencia de muestreo es de hasta 128 muestras por segundo y el diseño está conectado directamente al microcontrolador con comunicación I2C (Kusriyanto y Putra, 2018).

Sensor humedad del suelo FC-28: utiliza el concepto de resistencia en la conducción de electricidad a través del agua. El suelo húmedo conduce más debido a la presencia de más iones en el agua. El suelo seco conduce menos debido a la ausencia de contenido de agua. Por lo tanto, dependiendo del contenido de agua del suelo, el sensor puede mostrar un valor de hasta 1,023. Utiliza un voltaje de $3.3-5 \mathrm{~V}$, Corriente de 0.035 A y una señal de tipo analógica y digital. Tiene cuatro terminales que son las siguientes: VCC es poder, Ao es salida analógica, Do es salida digital y GND es tierra (Bhadani y Vashisht, 2019). 
Sensor de radiación ultravioleta UVM30A: capaz de detectar la radiación solar UV usando un circuito integrado UVM-30A. Puede detectar rayos UV con tamaño de onda entre $200-370 \mathrm{~nm}$ con rápida respuesta y control analógico. Su tensión de funcionamiento entre 3-5 V permite que sea fácilmente conectado a otros controladores (Ventura et al., 2020).

Sensor de lluvia FC37: este módulo se utiliza para averiguar si llueve. El módulo funciona midiendo la humedad obtenida de las gotas de agua recolectadas en la placa del sensor. La cantidad de gotas de agua hace que la resistencia paralela encontrada en la placa del sensor tenga un cambio de valor. Si las gotas de agua recolectadas en el suelo aumentan, el valor de resistencia que se convierte a la forma de voltaje será menor y si las gotas de agua recolectadas son pequeñas, el valor de voltaje cambia a alto. Cuando la placa del sensor está seca (no hay punto de agua), este módulo genera un voltaje analógico de $5 \mathrm{~V}$ (Kusriyanto y Putra, 2019).

Anemómetro: dispositivo que se utiliza para medir la velocidad del viento a la intemperie. Se compone de una carcasa, la copa de viento y el módulo de circuito. Los módulos fotovoltaicos, el procesador de microordenador industrial, el generador de corriente y la corriente eléctrica están integrados en el dispositivo (Chinaplccenter, 2019).

Módulo de reloj RTC DS3231: el tipo de reloj RTC (reloj en tiempo real), es un término empleado para especificar que se utiliza para medir el tiempo contabilizando pulsos de una señal, sin existir relación directa con unidades temporales. Tiene un voltaje de funcionamiento $3.3-5.5 \mathrm{~V}$ y su capacidad de almacenamiento es de 32K (Tahmidul et al., 2019).

Display GLCD ERM12864-2: recibe datos de 2,400 o 9,600 bits por segundo (bps) y muestra textos y gráficos. Se admiten dos fuentes: $8 \times 16$ píxeles (altura de caracteres de $8 \mathrm{~mm}$ ) y $6 \times 8$ píxeles $(4 \mathrm{~mm})$. Ambas fuentes son totalmente editables. Los G12864 tienen $16 \mathrm{~KB}$ de memoria flash no volátil utilizado para almacenar imágenes y fuentes (Scott Edwards Electronics, 2020).

\section{RESULTADOS}

Se realizó la captura y registro de las variables climáticas provenientes de las estaciones de campo y se analizaron datos comprendidos en el período de enero a diciembre 2019. En función de los registros diarios capturados por el sistema de monitoreo para la supervisión del clima en la producción de plátano, se pudo identificar las condiciones climáticas que se vieron afectadas por factores atmosféricos, las cuales se consideran fuera de rango como lo fueron los altos niveles humedad relativa, temperaturas altas y bajas en ciertas horas del día y altas precipitaciones, para lo cual se generaron alertas de forma remota que le permite al agricultor realizar estrategias de mitigación y adaptación del cultivo de plátano al cambio climático y prevenir daños severos ante eventos meteorológicos extremos.

\section{Temperatura}

La temperatura promedio es de $26^{\circ} \mathrm{C}$ siendo la temperatura máxima entre $29^{\circ} \mathrm{C}$ y $35^{\circ} \mathrm{C}$ con un promedio de $32^{\circ} \mathrm{C}$ y la temperatura mínima es entre $17^{\circ} \mathrm{C}$ y $23^{\circ} \mathrm{C}$ con un promedio de $20^{\circ} \mathrm{C}$. La Fig. 3 muestra que en los meses de junio (6) a agosto (8) la temperatura tiende a disminuir. La temperatura por encima de $37^{\circ} \mathrm{C}$ puede dañar la planta, pero crece bien bajo temperatura media de $27^{\circ} \mathrm{C}$ y dentro de $25^{\circ} \mathrm{C}$ a $30^{\circ} \mathrm{C}$ en un ambiente preferiblemente tropical, tierra baja húmeda (Salau et al., 2016).

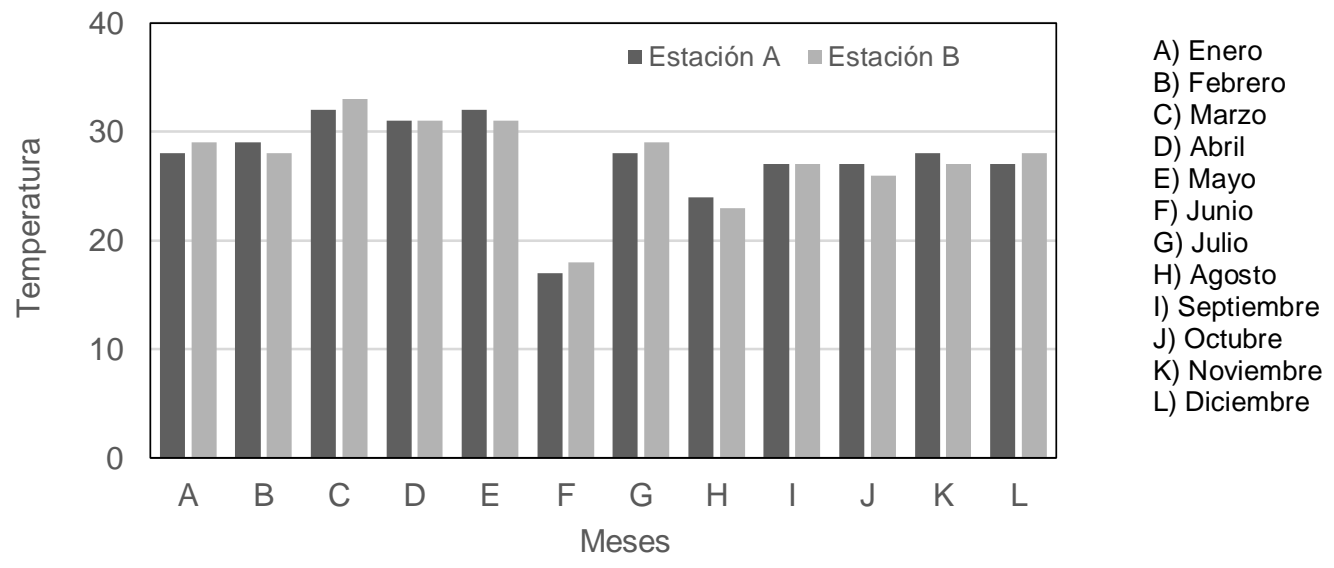

Fig. 3: Temperatura anual en el cultivo de plátano 


\section{Presión barométrica}

El cultivo se encuentra 6 metros sobre el nivel del mar. Se obtuvo un resultado de un promedio de $1,013.8$ milibares de presión por día, cuyos valores máximos se registraron en la mañana con 1,016.2 milibares en promedio y por las tardes un valor mínimo de 1,010.5 milibares. La presión barométrica es óptima para la producción del plátano en este cultivo. Se recomienda la siembra en zonas donde la altura varía de 0 a 300 metros sobre el nivel del mar. En el caso de suelos a nivel del mar, tomar en cuenta que no sean inundables y que tengan la capacidad de evacuación del agua (Vizcaíno et al., 2014).

\section{Radiación ultravioleta}

La Fig. 4 muestra que en los meses de junio a noviembre 2019, se obtuvo en promedio como valor máximo un nivel 7 de índex UV en horario de la tarde y valor mínimo registrado con un nivel 3 de índex UV en horarios de la madrugada. Si la radiación es baja $(<3)$, es más probable que se alcance la saturación de la luz de todas las hojas del plátano. A esto se le agrega la capacidad de las hojas de plátano para plegarse a medida que aumenta la radiación neta entrante, lo que resulta una energía radiante que se vierte para reducir los niveles de las hojas al suelo (Turner et al., 2007).

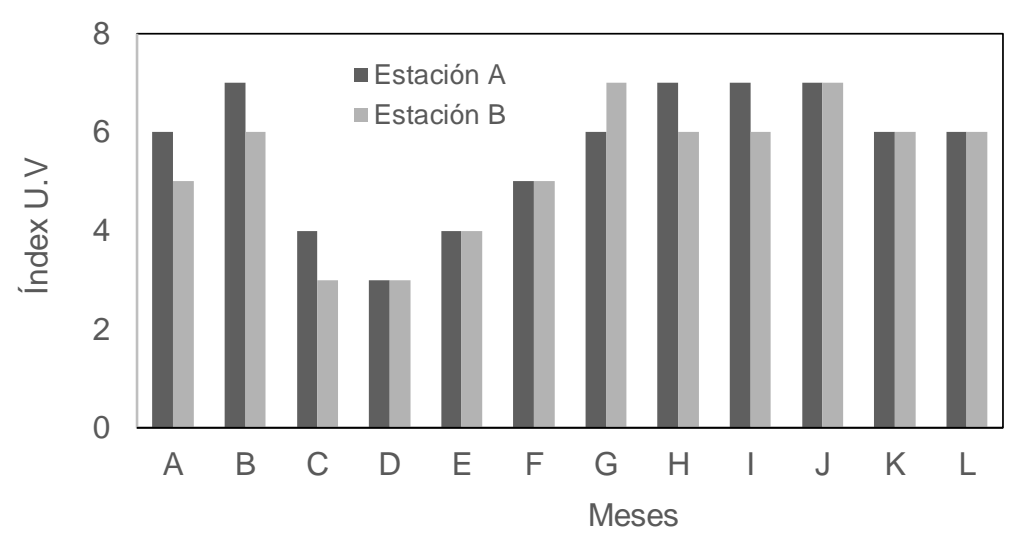
A) Enero
B) Febrero
C) Marzo
D) Abril
E) Mayo
F) Junio
G) Julio
H) Agosto
l) Septiembre
J) Octubre
K) Noviembre
L) Diciembre

Fig. 4: Radiación ultravioleta anual en el cultivo de plátano

\section{Pluviosidad}

Las lluvias se presentan en los meses de diciembre a mayo 2019, teniendo los meses de febrero y marzo 2019 , como los más lluviosos con precipitaciones superiores a los $280 \mathrm{~mm}$. La precipitación promedio anual es de $1,398 \mathrm{~mm}$. La época seca son los meses de junio a noviembre 2019, con una precipitación inferior a 30 mm. El plátano requiere una precipitación anual de aproximadamente 1,500 a 2,500 mm (Salau et al., 2016).

\section{Velocidad del viento}

El resultado promedio es de $3 \mathrm{~km} / \mathrm{h}$. Para lo cual por las tardes la velocidad de viento promedio es de $11 \mathrm{~km} / \mathrm{h}$ y el valor mínimo es en horarios de la madrugada con $0.1 \mathrm{~km} / \mathrm{h}$. La velocidad del viento superior a $15 \mathrm{~m} / \mathrm{s}$ pueden producir caída y arrancado de plantas, los vientos de menor intensidad (5-10 $\mathrm{m} / \mathrm{s})$ producen pérdidas de rendimiento (Galán y Robinson, 2013).

\section{DISCUSIÓN FINAL}

En el cultivo de plátano ubicado en Ecuador en la ciudad de Guayaquil cantón Durán, se pudo observar que existe un déficit hídrico significativo, de la misma manera la distribución de lluvias, ya que se presenta épocas de sequía severa y aproximadamente 120 días sin precipitaciones. El cultivo se puede beneficiar de la presencia de lluvias en épocas secas.

Gracias a este sistema se logró de forma exitosa, que el usuario pueda acceder a la información de su cultivo teniendo siempre la supervisión y vigilancia del cultivo de plátano vía remota. Además teniendo acceso a un historial de registros del clima y valores fuera de rango para la óptima producción del cultivo. De acuerdo a los resultados obtenidos, se puede identificar los factores del clima o eventos meteorológicos que afectan en el desarrollo fenológico de la planta de plátano, y con la generación de alarmas de forma remota se puede realizar correcciones tempranas más precisas al cultivo de plátano. Este estudio permite a los agricultores obtener una base para la planificación de estrategias de mitigación y adaptación al cambio climático, evaluando la idoneidad de los eventos meteorológicos que beneficien al cultivo en ciertas temporadas del año y prevenir daños severos ante eventos extremos. 
Comparando la tecnología empleada en el presente proyecto se utiliza componentes accesibles y económicos en el mercado tales como: XBee, SIM900, Arduino mega 2560, sensores tales como: temperatura, radiación ultravioleta, presión barométrica, pluviosidad y velocidad del viento, mientras que existen estaciones meteorológicas que son muy costosas, debido a su carácter comercial. Por ejemplo, según el estudio realizado por Ya'acob, et al. (2017), utilizan la plataforma IOT UBIDOTS, para un sistema de monitoreo ambiental, es decir envían 42 datos aproximadamente en un mes, mientras que en el presente trabajo a través del envío de mensajes de textos contratados por una operadora móvil, se puede enviar en una hora hasta 240 variables y se puede aumentar el número de envíos por hora, ya que trabaja con un plan ilimitado de mensajes (SMS). Adicional dicha información es registrada en una base de datos desarrollada en Qt Creator, que con una buena capacidad de almacenamiento de disco duro, no existiría ningún problema para guardar el histórico de datos.

Según el estudio realizado por Jiayu et al., (2015), existen tecnologías que trabajan orientadas a crear estaciones meteorológicas comerciales, estas cumplen la función específica de monitoreo de variables climatológicas solamente, mientras que nuestro sistema tiene la capacidad de fijar puntos críticos para la generación de alertas y el monitoreo del desarrollo del plátano, puede generar una señal de atención al operador en las variables de temperatura, radiación ultravioleta, presión barométrica, pluviosidad y velocidad del viento cuyos valores óptimos para la producción de plátano son: temperatura de $27^{\circ} \mathrm{C}$ a $30^{\circ} \mathrm{C}$ (Salau et al., 2016), radiación ultravioleta < 3 índex UV (Turner et al., 2007), presión barométrica con una altura de 0 a $300 \mathrm{mts}$ sobre el nivel del mar (Vizcaíno et al., 2014), pluviosidad de 1,500 a 2,500 mm anuales (Salau et al., 2016) y velocidad del viento de 10 a $15 \mathrm{~m} / \mathrm{s}$ (Galán y Robinson, 2013). Debido a lo expuesto anteriormente, se considera que el proyecto desarrollado en este trabajo resulta su implementación muy económica y rentable para los propietarios de los cultivos de plátanos que quieren monitorear el desarrollo de sus plantaciones y además recibir alertas cuando el crecimiento óptimo del plátano se encuentre sometido a peligros, donde los agricultores podrán tomar medidas correctivas instantáneas a ser ejecutadas en corto tiempo y así evitar pérdidas económicas y de producción. Todas estas bondades gracias a que el sistema diseñado tanto en hardware como en software es de libre acceso y por lo tanto de un costo económico.

\section{CONCLUSIONES}

De acuerdo al trabajo presentado y a los resultados obtenidos y su discusión, se pueden plantear las siguientes conclusiones: 1) El sistema de monitoreo desarrollado en este trabajo es rentable para los propietarios de los cultivos de plátanos debido a su bajo costo y a la implementación de hardware y software de libre acceso, brindando la facilidad de realizar monitoreo remoto del desarrollo de sus plantaciones, logrando facilitar un registro histórico de parámetros ambientales; 2) El sistema de alarmas indica cuando el crecimiento óptimo del plátano se encuentre sometido a peligros, permitiendo que el agricultor realice análisis y decisiones futuras o medidas correctivas instantáneas a ser ejecutadas en corto tiempo y así evitar pérdidas económicas y de producción, disminuyendo la cantidad de horas hombres empleadas en la producción; 3) La aplicación de tecnología inalámbrica posibilita la escalabilidad del sistema, ahorrando costos de instalación.

\section{REFERENCIAS}

Aurore, G., Parfait, B., y Fahrasmane, L., Bananas, raw materials for making processed food products, Trends Food Sci. Technol., ,20(2), 78-91 (2009)

Bhadani P. y Vashisht, V., Soil Moisture, Temperature and Humidity Measurement Using Arduino, 2019 9th International Conference on Cloud Computing, doi: 10.1109/CONFLUENCE.2019.8776973, Data Science \& Engineering (Confluence), 567-571 (2019).

Bhat, S., Kaul, N., Gupta, A., y Prakash, V., XBee Based Novel Wireless Technique to Simplify Locomotion of Physically Handicapped Persons, doi: 10.1109/SCES.2015.7506440, 2015 IEEE Students Conference on Engineering and Systems (SCES), 1-5 (2015)

Chinaplccenter, QS-FS Wind sensor, https://www.lollette.com/, acceso Marzo (2020)

Cintra W., Júnior D.J., Júnior R.V. y Cecílio R.A., Worldwide geographical distribution of Black Sigatoka for banana: Predictions based on climate change models, doi: 10.1590/S0103-90162008000700008, Sci Agric (Piracicaba, Braz), 65, 40-53 (2008)

Galán, V. y Robinson, J., Fisiología, Clima y Producción de Banano, XX Reunião Internacional da Associação para a Cooperação em Pesquisa e Desenvolvimento Integral das Musáceas (Bananas e Plátanos), Fortaleza, Brasil (2013).

Granato, D., de Magalhães, M., Fogliano, V., y van Ruth, S. M., Effects of geographical origin, varietal and farming system on the chemical composition and functional properties of purple grape juices: A review, doi: 10.1016/j.tifs.2016.03.013, Trends Food Sci. Technol., 52, 31-48 (2016) 
Jiayu, Z., Shiwei, X., Zhemin, L, Wei, C, y Dongjie, W., Application of Intelligence Information Fusion Technology in Agriculture Monitoring and Early-warning Research, doi: 10.1109/ICCAR.2015.7166013, 2015 International Conference on Control, Automation and Robotics, 114-117 (2015)

Khan, R., Ali, I., Zakarya, M. y otros tres autores, Technology-Assisted Decision Support System for Efficient Water Utilization: A Real-Time Testbed for Irrigation Using Wireless Sensor Networks, doi: 10.1109/ACCESS.2018.2836185, IEEE Access, 6, 25686-25697 (2018)

Kusriyanto, M. y Putra, A.A., Weather Station Design Using loT Platform Based On Arduino Mega, doi: 10.1109/l, SESD.2018.8605456, 2018 International Symposium on Electronics and Smart Devices (ISESD), 1-4 (2018)

Luna, M.L., Becerra, S.M., Serrano, N. y Lobo, R.A., Implementación de tecnologías libres y sensores remotos para un biobanco: el desafío de producir a bajo costo, doi: 10.4067/S0718-07642020000200149, Inf. Tecnol. 31(2), 149-162 (2020)

Machovina, B. y Feeley, K.J., Climate change driven shifts in the extent and location of areas suitable for export banana production, doi: 10.1016/j.ecolecon.2013.08.004, Ecol. Econ. , 95, 83-95 (2013)

Muñoz, Y.Y., Castrillón, O.D., Castillo, L.F. y López, C., Análisis de la Escena en la Cocina por Medio de Sensores loT Diseñados Basados en el Microcontrolador Node MCU ESP8266 y Conectados al Servidor ThingSpeak, doi: 10.4067/S0718-07642019000500173, Inf. Tecnol. 30(5), 173-190 (2019)

Nelson, G.C., Rosegrant M.W., y otros doce autores, Climate change: Impact on agriculture and costs of adaptation, doi: 10.2499/08962953, Food Policy Report, Washington (2009)

Poudel, S. y Kotani, K., Climatic impacts on crop yield and its variability in Nepal: Do they vary across seasons and altitudes?, doi: 10.1007/s10584-012-0491-8, Clim. Change, 116, 327-355 (2013)

Rafiq, A.A., Yusuf, M. y Pujono, Implementation of Digital Image Processing Using NI myRIO and Arduino Mega 2560 as Controller On Rover Bogie Robot, doi: 10.1109/iCAST1.2018.8751506, 2018 International Conference on Applied Science and Technology (iCAST), 210-215 (2018)

Sadowski, S. y Spachos, P., Wireless technologies for smart agricultural monitoring using internet of things devices with energy harvesting capabilities, doi: 10.1016/j.compag.2020.105338 , Comput. Electron. Agric., 172 (2020)

Salau, O.R., Momoh, M., Olaleye, O.A. y Owoeye, R.S., Effects of Changes in Temperature, Rainfall and Relative Humidity on Banana Production in Ondo State, Nigeria, World Sci. News, 44, 143-154 (2016)

Scott Edwards Electronics, User's Manual 128x64-pixel Serial Graphics LCD (G12864 v2.0), https://www.seetron.com/, acceso Marzo (2020)

Singh, B., Singh, J. P., Kaur, A., y Singh, N., Bioactive compounds in banana and their associated health benefits - A review, doi: 10.1016/j.foodchem.2016.03.033, Food Chem. , 206, 1-11 (2016)

Soares, J.D.R., Pasqual, M. y tres autores, Utilization of artificial neural networks in the prediction of the bunches' weight in banana plants, doi: 10.1016/j.scienta.2013.01.026, Sci. Hortic., 155, 24-29 (2013)

Sujakhu, N.M., Ranjitkar, S. y otros cuatro autores, Farmers' Perceptions of and Adaptations to Changing Climate in the Melamchi Valley of Nepal, doi: 10.1659/MRD-JOURNAL-D-15-00032.1, Mt. Res. Dev. , 36(1), 15-30 (2016)

Tahmidul, A.Z.M., Deb, N. y tres autores, Six Tier Multipurpose Security Locker System Based on Arduino, doi: 10.1109/ICASERT.2019.8934615, 2019 1st International Conference on Advances in Science, Engineering and Robotics Technology (ICASERT), 1-5 (2019)

Turner, D.W., Fortescue, J.A. y Thomas, D.S., Environmental physiology of the bananas (Musa spp.), doi: 10.1590/S167704202007000400013, Braz. J. Plant Physiol., 19(4), 463-484 (2007)

Van Rijn, F., Fort, R., y otros tres autores, Does certification improve hired labour conditions and wageworker conditions at banana plantations?, doi: 10.1007/s10460-019-09990-7, Agr. Hum. Values, (2019)

Ventura, V., Sensor de radiación ultravioleta con Arduino, https://polaridad.es/ acceso Marzo (2020)

Vizcaíno, D.A., Betancourt, R.A. y otros cinco autores, Guía de buenas prácticas agrícolas para banano, Agencia Ecuatoriana de Aseguramiento de Calidad del Agro- Agrocalidad, Ecuador (2014)

Wang, Z., Erasmus, S.W. y otros cuatro autores, Linking growing conditions to stable isotope ratios and elemental compositions of Costa Rican bananas (Musa spp.), doi: 0.1016/j.foodres.2019.108882, Food Res. Int., 129 (2020)

Ya'acob, N., Zolkapli, M., y otros cuatro autores, UAV Environment Monitoring System, doi: 10.1109/ICEESE.2017.8298395, 2017 International Conference on Electrical, Electronics and System Engineering (ICEESE), 105-109 (2017) 